Malignant eccrine spiradenoma is an extremely rare sweat-gland tumour. Herein we aimed to present an 81-yearold woman with malignant eccrine spiradenoma repeated on the thigh and metachronous lung cancer. She presented with a palpable swelling in the right thigh region. Her systemic screening was normal and excisional biopsy was performed. A diagnosis of malignant eccrine spiradenoma was made. Thirty-two months later, the tumour recurred in the same region. The tumour was excised again. Lymph node metastasis was also detected in lymph node dissection. Fifteen months later, the tumour recurred again in the same place. In addition, lymph node metastasis and bone metastasis were found. Palliative radiotherapy was performed for the involvement of the right femur. The treatment for metachronous lung cancer could not be given because of the patient's age and performance, and she could not be given additional treatment for the same reasons.

Key words: de novo, malignant eccrine spiradenoma, metachronous lung cancer, palliative radiotherapy.

Contemp Oncol (Pozn) 2014; 18 special issue DOI: $10.5114 /$ wo.2014.40598

\section{De novo malignant eccrine spiradenoma and metachronous lung cancer}

Dilek Unal ${ }^{1}$, Arzu Tasdemir ${ }^{2}$, Arzu Oguz ${ }^{3}$, Ali Koc ${ }^{4}$, Yasemin Cihan Benderli5

1Department of Radiation Oncology, Erciyes University Medical Faculty 2Department of Pathology, Kayseri Education and Research Hospital, Kayseri, Turkey ${ }^{3}$ Department of Medical Oncology, Kayseri Education and Research Hospital, Kayseri, Turkey

${ }^{4}$ Department of Radiology, Kayseri Education and Research Hospital, Kayseri, Turkey ${ }^{5}$ Department of Radiation Oncology, Kayseri Education and Research Hospital, Kayseri, Turkey

\section{Introduction}

Eccrine spiradenoma is a benign tumour of the sweat glands characterized by its slow growth and long clinical course [1]. It generally occurs as a solitary intradermal nodule measuring 1-2 cm in diameter, arises in early adulthood and has no characteristic location. The nodules are often tender and occasionally painful [2]. Malignant eccrine spiradenoma (MES) is one of the extremely rare sweat gland tumours and often arises from benign eccrine spiradenoma or, less commonly, may develop de novo [1, 3]. Transformation into malignant form generally occurs 20 to 30 years after initial detection, with a latency period ranging from 6 to 70 years [3-5]. Malignant eccrine spiradenoma occurs predominantly in older patients regardless of the ethnic group, with a slight female predominance [4, 5]. It sometimes develops in young patients [6, 7]. Herein we aimed to present an 81-year-old woman with MES repeated on the thigh and metachronous lung cancer.

\section{Case report}

An 81-year-old female patient presented with a palpable painful swelling on the right groin. Physical examination revealed that a non-ulcerated mobile mass approximately $6 \mathrm{~cm}$ in size on the inner side of the right thigh. The patient's systemic scan was normal and excisional biopsy was performed. The pathology findings were compatible with MES (Fig. 1). The patient did not attend any follow-up examinations. Thirty-two months later, she again presented with swelling in the same place. Excisional biopsy and lymph node dissection were performed. Pathologic evaluation revealed the same tumour. Four metastatic lymph nodes were detected (Fig. 2). There were close surgical margins (approximately $1 \mathrm{~mm}$ ). She again failed to attend any follow-up examinations. Fifteen months later, she again presented with swelling in the same place. Abdominal computerized tomography (CT) scan revealed bilateral para-aortic and para-iliac metastatic lymph nodes. She had undergone whole-body bone scintigraphy due to leg pain. Suspected involvement of the right femur was determined. Positron emission tomography detected right inguinal mass (Fig. 3A), widespread metastatic lymph nodes and a lung mass approximately $98 \mathrm{~mm} \times 90 \mathrm{~mm}$ in size (Fig. 3B), which was considered as a primary malignant process. Transthoracic lung biopsy was reported as malignant tumour, which did not resemble MES. Due to the radiological and pathological findings, it was thought to be a second primary lung cancer. The patient refused the second biopsy recommended for a definitive diagnosis. We were unable to verify the origin of the right inguinal mass and metastatic lymph nodes. Biopsy was needed to definitive- 


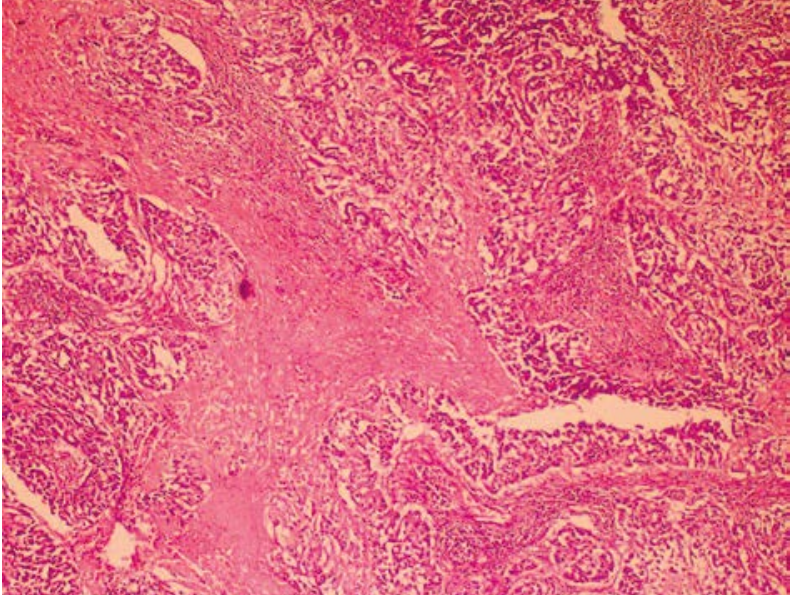

Fig. 1. Marked infiltration of the tumour with focal areas of necrosis (haematoxylin and eosin staining, magnification 10x)

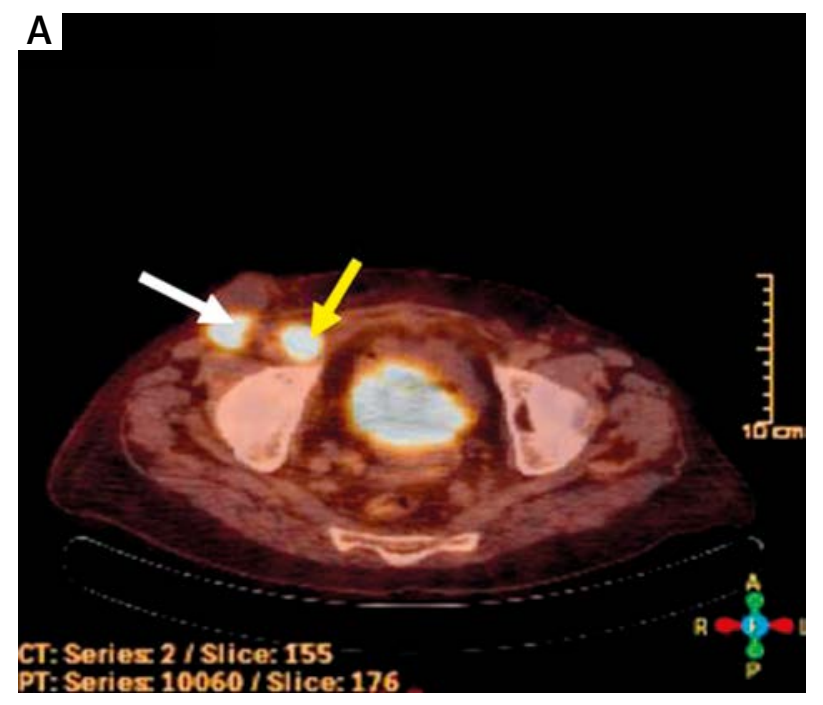

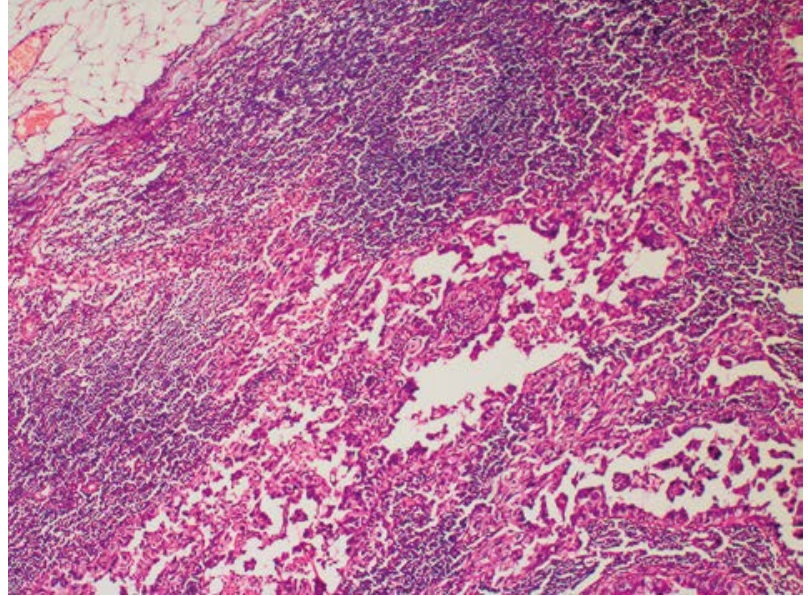

Fig. 2. Metastasis to lymph nodes of the tumour (haematoxylin and eosin staining, magnification 10x)

Fig. 3. A) white arrowhead shows inguinal mass, whereas yellow arrowhead shows metastatic lymph node in positron emission tomography, B) arrowhead shows lung mass

ly establish the origin of the mass and these metastases. However, biopsy was not performed because she refused all invasive procedures; therefore, we could not obtain definitive evidence, but we think that the origin of the mass and these metastases may have been MES or lung cancer. Palliative radiotherapy was performed for femur bone metastasis and lesions in the inguinal region at a total dose of 3000 cGy (300 cGy/day; 5 days/week/10 fractions). Additional treatment could not be applied to the patient due to her age and performance.

\section{Discussion}

Eccrine spiradenomas are benign adnexal tumours with slow growth and a long clinical course [8]. The imaging findings of eccrine spiradenomas have been demonstrated in only a few cases. The ultrasonography findings of an eccrine spiradenoma in the upper arm were reported by Jin et al. [9]. Ultrasonography revealed a well-defined lobulating mass with heterogeneous hypoechogenicity in the deep portion of the dermis and superficial subcuta- neous fat layer without connection of the epidermis and extension into any muscular structures. Focally, more hypoechoic portions were detected in the mass [9]. The magnetic resonance (MR) images of multiple eccrine spiradenomas were reported by Han et al. [10]. Magnetic resonance revealed extensive nodules with low signal intensity on T1-weighted images and high signal intensity on the short $\mathrm{T} 1$ inversion recovery images in the cutis and subcutaneous tissue. The lesions, scattered, with some of them clustered, were separated from each other with relatively clear margination [10].

Malignant eccrine spiradenoma is an extremely rare tumour and usually originates from benign eccrine spiradenoma, but sometimes develops de novo $[3,11]$. In the present patient, there was no history of benign eccrine spiradenoma before the development of malignant tumour. The tumour was thought to have developed de novo.

The typical clinical manifestation of MES is a chronic skin mass with recent rapid growth, which may show erythema, ulceration, bleeding and satellite nodules [3]. Malignant eccrine spiradenoma may occur in any location 
with a slight predominance in the trunk and extremities $[1,11]$. The tumour usually develops in older patients, with minimal gender predilection [11]. Similarly, the clinical presentation in our patient was a palpable mass on the thigh, and the patient was in her eighties.

Histopathological examination gives the diagnosis of most patients with MES. The classic histopathology of MES is solid islands of anaplastic basaloid cells arranged in cords and nodules, with highly pleomorphic cells containing abundant mitotic figures. These cells may express cytokeratins, epithelial membrane antigen (EMA), p53 and S100. Malignant transformation usually includes invasion of tumour cells into adjacent stroma $[3,4]$. In the present patient, immunohistochemical analysis of biopsy specimens showed a positive immunohistochemical staining with keratin, actin, EMA (Fig. 4) and S100. In addition, infiltration into surrounding stroma was observed.

Malignant eccrine spiradenoma is an aggressive sweat gland tumour with poorly understood behaviour [3]. The prognosis of the tumour is variable depending on whether there is lymph node involvement or distant organ metastasis. Andreoli et al. reported on a meta-analysis of 72 reported cases with MES [3]. In 35 patients without metastatic disease, local resection resulted in 100\% disease-free survival. All 12 patients, who had lymph node involvement without distant organ metastasis, underwent surgical resection of the tumour, with 7 patients receiving additional lymph node dissection. Of the total 12 patients, 3 without lymph node dissection died due to metastatic disease (45 months of mean survival), 1 patient was alive with disease 24 months after lymph node dissection and 8 patients lacked evidence of recurrent disease in the final follow-up evaluation. In 24 patients with distant organ metastasis, median survival was 16 months [3]. Similarly, in a paper reported by Tanese et al., all patients with distant organ metastasis died, whereas all cases without metastasis were alive [4].

Recurrence does not occur in approximately two thirds of patients with MES. Hantash et al. reported a case report and review of the literature. In their report, 45 of the $96 \mathrm{pa}$ tients with MES had no recurrence, whereas it developed in 32 patients. On the other hand, 19 had no data available about recurrence [5]. Similarly, in a paper including 16 Japanese patients with MES, recurrence did not occur in most of these cases [4].

A definitive diagnosis of metastasizing MES is made only when a biopsy of a metastasis is shown to be similar to the primary skin lesion [8]. We were unable to verify the origin of the right inguinal mass and metastatic lymph nodes because biopsy was not performed. Therefore, we could not obtain definitive evidence, but we think that the origin of the mass and these metastases may have been MES or lung cancer.

Because there are a limited number of case reports on the treatment of MES in literature, the treatment is not evidence based. In most cases, the primary treatment modality is wide local excision. Lymph node dissection is an effective method of treatment in the presence of lymph node metastases. Non-surgical treatments, including chemotherapy, radiotherapy and hormonal therapies, remain

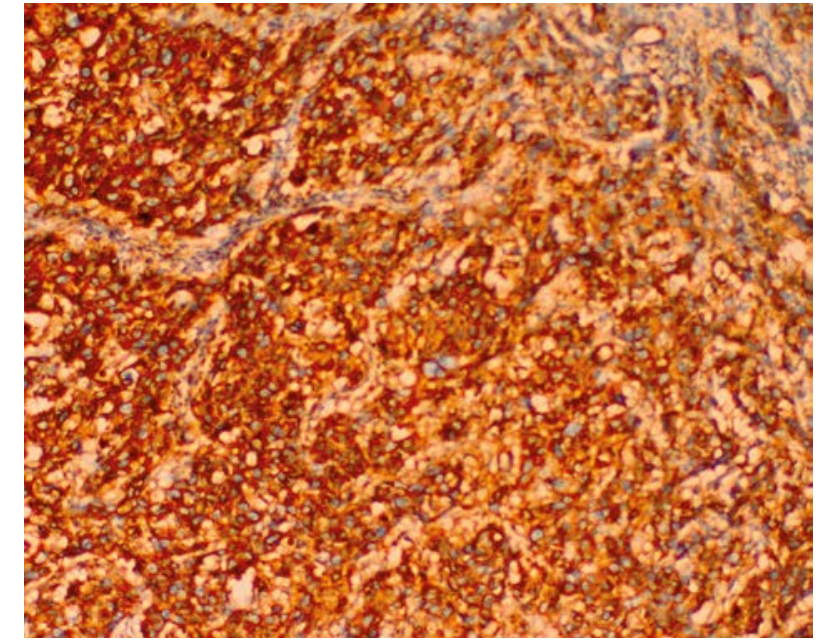

Fig. 4. Positive immunohistochemical staining with epithelial membrane antigen (magnification 10x)

limited with only anecdotal evidence to support their efficacy [5]. Therefore, treatment of patients with visceral metastases is difficult, and mortality in these patients is extremely high. Radiotherapy, alone or in combination with chemotherapy, is rarely successful in treating metastatic MES [2, 4]. It is generally known that sweat gland tumours are radioresistant and there is little role of radiotherapy in the treatment of such tumours [11, 12]. Data about chemotherapy is very limited because the tumours are very rare. In patients with metastatic disease, chemotherapeutic agents such as 5-fluorouracil, epirubicin, mitomycin, vincristine and carboplatin were used, but no successful response was achieved [2]. It was reported that there were improvements with tamoxifen therapy in cases with oestrogen receptor-positive MES [11, 13]. In the present patient, excision of the primary tumour and lymph node dissection was the form of treatment choice in the presentation and the first relapse. On the other hand, when widespread disease developed, further treatment was not considered except for local radiotherapy to the bone lesion. Tamoxifen therapy was not thought to be oestrogen receptor-negative.

Multiple malignancies in the same patient account for $1.84 \%$ to $3.9 \%$ of all cancers [14]. Second primary tumours are encountered with increasing frequency in recent oncology clinical practice. Metachronous second primary tumours are diagnosed more than six months after the first primary tumour, while synchronous second primary tumours are diagnosed within six months of the first primary tumour. In the present patient, lung cancer probably developed as the second primary cancer and appeared to be more important than the malignant eccrine spiradenoma in determining the prognosis.

In conclusion, MES, which develops de novo in elderly patients, may have a better prognosis than expected. With proper treatment, survival rates can be increased. In addition, metachronous cancers such as lung cancer may develop in the follow-up of MES.

Authors declare no conflict of interest. 


\section{References}

1. Ben Brahim E, Sfia M, Tangour M, Makhlouf R, Cribier B, Chatti S. Malignant eccrine spiradenoma: a new case report. J Cutan Pathol 2010; 37: 478-81.

2. Ishikawa M, Nakanishi Y, Yamazaki N, Yamamoto A. Malignant eccrine spiradenoma: a case report and review of the literature. Dermatol Surg 2001; 27: 67-70.

3. Andreoli MT, Itani KM. Malignant eccrine spiradenoma: a meta-analysis of reported cases. Am J Surg 2011; 201: 695-9.

4. Tanese K, Sato T, Ishiko A. Malignant eccrine spiradenoma: case report and review of the literature, including 15 Japanese cases. Clin Exp Dermatol 2010; 35: 51-5.

5. Hantash BM, Chan JL, Egbert BM, Gladstone HB. De novo malignant eccrine spiradenoma: a case report and review of the literature. Dermatol Surg 2006; 32: 1189-98.

6. Varsa EW, Jordan SW. Fine needle aspiration cytology of malignant spiradenoma arising in congenital eccrine spiradenoma. Acta Cytol 1990; 34: 275-7.

7. Zamboni AC, Zamboni WA, Ross DS. Malignant eccrine spiradenoma of the hand. J Surg Oncol 1990; 43: 131-3.

8. Leonard N, Smith D, McNamara P. Low-grade malignant eccrine spiradenoma with systemic metastases. Am J Dermatopathol 2003; 25: 253-5.

9. Jin W, Kim GY, Lew BL, Yang DM, Kim HC, Ryu JK, Park JS, Ryu KN. Sonographic findings of an eccrine spiradenoma: case report and literature review. J Ultrasound Med 2008; 27: 813-8.

10. Han YD, Huan Y, Deng JL, Zhang YG, Zhang CH. MRI appearance of multiple eccrine spiradenoma. Br J Radiol 2007; 80: e27-9.

11. Mirza I, Kloss R, Sieber SC. Malignant eccrine spiradenoma. Arch Pathol Lab Med 2002; 126: 591-4.

12. Morris DM, Sanusi ID, Lanehart WH. Carcinoma of eccrine sweat gland: experience with chemotherapy, autopsy findings in a patient with metastatic eccrine carcinoma, and a review of the literature. J Surg Oncol 1986; 31: 26-30.

13. Sridhar KS, Benedetto P, Otrakji CL, Charyulu KK. Response of eccrine adenocarcinoma to tamoxifen. Cancer 1989; 64: 366-70.

14. Balat O, Kudelka AP, Ro JY, Edwards CL, Balbay D, Dinney C, Kavanagh JJ. Two synchronous primary tumors of the ovary and kidney: a case report. Eur J Gynaecol Oncol 1996; 17: 257-9.

\section{Address for correspondence}

Dilek Unal MD, PhD

Department of Radiation Oncology

Erciyes University Medical Faculty

38039 Kayseri, Turkey

e-mail: dilekunaldr@gmail.com

Submitted: 20.11.2012

Accepted: $\quad 5.02 .2013$ 